\title{
Modelo de valoración con opciones reales, rejillas trinomial, volatilidad cambiante, sesgo y función isoelástica de utilidad
}

\author{
MILANESI, GASTÓN \\ Universidad Nacional del Sur (Argentina) \\ Correo electrónico: milanesi@uns.edu.ar
}

\begin{abstract}
RESUMEN
La valoración de inversiones en empresas de base tecnológica, intangibles y startup en mercados financieros emergentes, imperfectos e incompletos, tornan cuestionable el tradicional enfoque binomial de opciones reales. Por ello se propone un modelo numérico que modifica al tradicional modelo binomial, incorporando rejillas trinomiales, volatilidad cambiante, funciones isoelásticas de utilidad y aversión al riesgo variable. Estas características pretenden mejorar la valoración de proyectos no convencionales en mercados emergentes. Se empleó el método de análisis de casos para administración analizando la valoración de una estrategia de inversión sobre una firma de base tecnológica. Los resultados obtenidos permiten comparar los diferentes valores, desde el clásico modelo binomial hasta el modelo numérico propuesto. Este último demostró superioridad, debido a que incorpora explícitamente variables en el proceso de valoración, como las preferencias del inversor frente al riesgo y los niveles de volatilidad según el ciclo de vida.
\end{abstract}

Palabras clave: opciones reales, trinomial, volatilidad cambiante, funciones isoelásticas de utilidad, aversión variable al riesgo, valuación start-up.

Clasificación JEL: G13; G31.

MSC2010: 91G50. 


\title{
Valuation model with real options, trinomial lattice, changing volatility, bias and isoelastic utility functions
}

\begin{abstract}
At emerging financial markets, the $R \& D$, intangible and technological basis firms (TBF) valuation, they make the traditional real option binomial approach questionable. For that, a numerical model that modified the traditional binomial model is proposed, incorporating trinomial lattice, changing volatility, isoelastic utility function and variable risk aversion. These characteristics pretend improve the no conventional project valuation in emerging markets. It is employed the case method of analysis in administration, analysing the investment strategy valuation over a technological basis firm. The obtained results allow to compare the different values, from the classical binomial model until the proposed numerical model. The last showed superiority, because its incorporates explicitly variables in the valuation process, like the investor preference for risk and volatility levels according the life cycle.
\end{abstract}

Keywords: real options, trinomial, changing volatility, isoelastic utility functions, variable risk aversion, start-up valuation.

JEL classification: G13; G31.

MSC2010: 91G50.

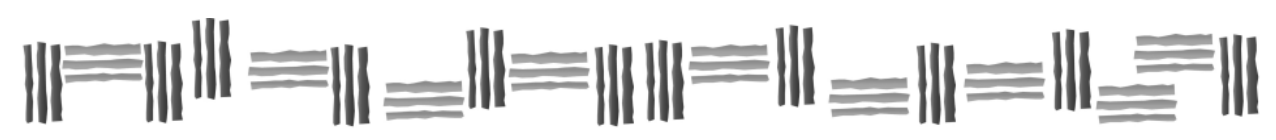




\section{Introducción.}

Un proceso de valoración implica una actividad intelectual donde se debe tomar conocimiento del activo real objeto de valoración, con el fin de generar información cualitativa y cuantitativa para formar juicios de valor; éstos son sintetizados en una medida de valor. Éste es una expresión subjetiva que refleja expectativas futuras cuantitativas relativas a la corriente de beneficios futuros del activo. Tal subjetividad cede en el momento en el que las partes acuerdan un precio de transferencia. Por lo tanto, el precio contiene expectativas de valor, vinculadas al espacio temporal donde se perfeccionó la transacción. Existen múltiples modelos para valorar activos reales, siendo los modelos de opciones reales los que mejor se adaptan para valorar la flexibilidad estratégica del proyecto (Smith \& Nau, 1995; Trigeorgis, 1995, 1997; Amram \& Kulatilaka, 1998; Copeland \& Antikarov, 2001; Smit \&Trigeorgis, 2004; Shockley, 2006; Graeme, 2009; Brandao \& Dyer, 2009; Num, 2015; Salahaldin, 2016, entre otros). Una tipología particular la componen los proyectos de inversión de base tecnológica desarrollados en mercado emergentes. En estos casos, el desafío de valoración y del modelo a utilizar incrementa su complejidad en virtud a las características del proyecto dadas por: (i) ambigüedad y/o inexistencia de información comparable o de mercado sobre los riesgos asociados a los flujos de la nueva inversión, (ii) complejidad de la inversión, dada por sus múltiples fuentes de opcionalidad, volatilidades cambiantes a lo largo de su ciclo de vida y sesgos en su valor, (iii) preferencias o grado de aversión al riesgo del inversor, quien por restricciones del medio, no puede replicar riesgos del proyecto con carteras de cobertura, frente a mercados no completos.

El trabajo propone un modelo numérico de valoración de empresas de base tecnológicas, intangibles y estrategias en I\&D entre otras, que considere las complejidades planteadas precedentemente. Reconoce sus principales cimientos en trabajos que brinda respuesta parcial a los desafíos planteados (Haahtela, 2010a, 2010b; Milanesi, Pesce \& El Alabi, 2014; Ochoa \&Vasseur, 2014; Pareja \& Cadavid, 2016; Pareja \& Baena, 2018; Milanesi, 2018). El modelo reúne el empleo de rejillas trinomiales, adaptando los niveles de volatilidad al ciclo de vida del proyecto e incorporando sesgos en la distribución de probabilidad de valor. Asimismo, utiliza funciones exponenciales isoelásticas de utilidad, con grados cambiantes de aversión al riesgo en el afán de representar la percepción de valor del agente en mercados imperfectos.

La estructura del trabajo es la siguiente. A continuación, es desarrollado formalmente el modelo: rejillas trinomiales, sesgos en la proyección del proceso estocástico, volatilidades cambiantes según el ciclo de vida, nuevos coeficienes equivalentes ciertos y el uso de funciones isoelásticas de utilidad en el proceso recursivo de valoración. En la tercera sección se analiza el caso de una EBT con opción de transferencia o expansión. El modelo es sometido a un análisis de sensibilidad bivariado relativo al sesgo y amplitud de movimientos en cada nodo de la rejilla. Son comparados los resultados obtenidos con el modelo binomial clásico, trinomial clásico, trinomial clásico con volatilidad cambiante, trinomial con volatilidad cambiante y sesgo. Finalmente, se concluye sobre las ventajas del modelo numérico propuesto, puesto que su estructura permite incorporar los elementos que hacen a la complejidad del activo: flexibilidad estratégica, ambigüedad de datos, sesgo en su valor, riesgos cambiantes y sujetos no neutrales al riesgo.

\section{El modelo trinomial con volatilidad cambiante, desplazamiento y funciones isoelásticas de utilidad.}

En esta sección serán desarrollados los pilares del modelo numérico de valoración propuesto, comenzando con sus fundamentos en las rejillas binomiales hacia las rejillas trinomiales, la incorporación de volatilidad cambiante, sesgo en el valor esperado del subyacente y el uso de funciones isoelásticas exponenciales de utilidad. 


\subsection{Rejillas binomiales.}

Las rejillas son herramientas empleadas para proyectar el valor del activo subyacente y valorar opciones. Éstas presentan ventajas computacionales en relación a los árboles de decisión debido a sus propiedades recombinantes de nodos intermedios (Smith, 2005). La propiedad de recombinación de las rejillas asegura que en el paso $\mathrm{N}$ existe $\mathrm{N}+1$ nodos finales y $\mathrm{n}(\mathrm{n}+1) / 2$ puntos de decisión mientras que en los árboles binomiales la cantidad de nodos finales asciende a $2 n-1$ y los puntos de decisión a 2n-1.

Se caracterizan por modelar en tiempo discreto el proceso estocástico continuo de la variable y utilizar el supuesto de valoración neutral al riesgo (Wilmott, 2009). Se basa en la perfecta correlación entre los cambios en el valor de la opción y del activo subyacente. Una cartera compuesta por una posición larga (corta) en una opción y corta (larga) en el subyacente hace que un activo de alto riesgo cubra las fluctuaciones en el precio del derivado. El valor de la cartera crece al tipo sin riesgo y el número exacto de subyacente a vender (comprar) se conoce como "delta". En un mundo del tipo "BlackScholes" si se compran "delta" acciones empleando el modelo, entonces se cubre correctamente el riesgo eliminándolo por completo.

El modelo binomial se caracteriza porque el activo subyacente presente movimiento de ascenso $(u)$ y descenso $(d)$ para un intervalo de tiempo discreto $(\Delta t)$ con probabilidad de transición $p$ y $1-p$. Los parámetros del modelo son $p$, $u$, y $d$, con la condición $p_{u}+p_{u}=1 ; 0<p<1$. La otra restricción en las rejillas es la condición de recombinación, $u . d=d \cdot u=m^{2}$.

Quizá el modelo más difundido es el denominado binomial CRR (Cox, Ross \& Rubinstein, 1979). Sus parámetros son:

$$
\begin{aligned}
& u=e^{\sigma \sqrt{ } \Delta t} \\
& d=e^{-\sigma \sqrt{ } \Delta t} \\
& p=\frac{1}{2}+\frac{1}{2}\left(\frac{\mu}{\sigma}\right) \sqrt{\Delta t}
\end{aligned}
$$

La expresión tradicional correspondiente a las probabilidades de transición (ecuación 3) es (Whaley, 2006):

$$
p=\frac{e^{r \Delta t}-d}{u-d}
$$

Alternativamente se encuentra el modelo binomial de Rendleman y Bartter (1979) y Jarrow y Rudd (1982). En este modelo se sostiene que la mejor aproximación al valor esperado y media del proceso browniano ocurre si $\mu=r-\sigma^{2} / 2$, derivando en probabilidades de transición con valor $p=$

$1 / 2$ y parámetros $u=e^{\left(r-\frac{\sigma^{2}}{2}\right) \Delta t+\sigma \sqrt{ } \Delta t} ; d=e^{\left(r-\frac{\sigma^{2}}{2}\right) \Delta t-\sigma \sqrt{ } \Delta t}$ y $u . d=e^{2\left(r-\frac{\sigma^{2}}{2}\right) \Delta t} \quad$ (Jabbour, Kramin $\&$ Young, 2001). La propiedad que las probabilidades neutrales al riesgo deben ser $1 / 2$ se atribuye a Jarrow y Rudd (1982). Asimismo, cabe destacar que existe una amplia variedad y gamas de formulación relacionadas con el modelo binomial (Van der Hoek \& Elliot, 2006; Chance, 2007).

\subsection{Rejillas trinomiales.}

Las rejillas trinomiales contienen en su estructura una mayor cantidad de movimientos futuros del subyacente, siendo tres movimientos por nodo $(u, m y d)$. Consecuentemente convergen con mayor velocidad a los resultados generados por el modelo continuo. Las premisas a seguir para su construcción son similares a las condiciones exigidas para las rejillas binomiales (Boyle, 1988; Kamrad \& Ritchken, 1991; Tian, 1993; Derman, Kani \& Chriss, 1996; Van der Hoek \& Elliot, 2006; Whaley, 2006; Chance, 
2007; Guthrie, 2011; Hull, 2012). En efecto, éstas son: (i) probabilidades de transición positivas en el límite entre 0 y 1 debiendo ser su suma equivalente a la unidad: $p_{u}+p_{m}+p_{d}=1$; (ii) la media $(M)$ y varianza $(V)$ de la distribución trinomial debe ser igual a los primeros dos momentos estocásticos de la distribución lognormal: (media) $p_{u} S_{u}+p_{m} S_{m}+p_{d} S_{d}=M$; (varianza) $p_{u}\left(S^{2} u^{2}-S^{2} M^{2}\right)+$ $p_{m}\left(S^{2} m^{2}-S^{2} M^{2}\right)+p_{d}\left(S^{2} d^{2}-S^{2} M^{2}\right)=S^{2} M^{2} V$, donde $M=e^{r \Delta t}$ y $V=e^{\sigma^{2} \Delta t}-1$.

Conforme fue analizado, el modelo binomial plantea dos parámetros: $u, d$ y $m=1$, ya que $m=u . d$. Para las rejillas trinomiales, Boyle (1988) y Kamrad y Ritchken (1991) plantean un valor $\lambda$ que regula la amplitud de los movimientos laterales de manera directamente proporcional. El valor de $\lambda$ es directamente proporcional a la amplitud de movimientos. A mayor valor del coeficiente, mayor la probabilidad de movimientos laterales, siendo $\lambda \geq 1$. El coeficiente de ascenso tiene la forma funcional $u=e^{\lambda \sigma \sqrt{ } \Delta t}, \quad d=e^{\lambda-\sigma \backslash \Delta t}$ y $m=u . d$. Fijando $u . d=1$ y sujeto a las condiciones de media y varianza, Boyle plantea las probabilidades de transición como:

$$
\begin{aligned}
& p_{u}=\frac{\left(V+M^{2}-M\right) u-(M-1)}{(u-1)\left(u^{2}-1\right)} \\
& p_{d}=\frac{u^{2}\left(V+M^{2}-M\right)-u^{3}(M-1)}{(u-1)\left(u^{2}-1\right)} \\
& p_{m}=1-p_{u}-p_{d}
\end{aligned}
$$

Cuando el coeficiente $\lambda$ es igual a 1 el modelo arroja similares resultados que el modelo binomial. En este caso la probabilidad de no cambio en el precio es igual a 0 provocando la eliminación de los nodos del medio. El coeficiente de ascenso se resume a, $u=e^{\lambda \sigma \Delta \Delta t}=e^{\sigma \vee \Delta t}$ y las probabilidades de movimientos ascendentes y descendentes quedan iguales a las del modelo de Cox, Ross y Rubinstein (1979). Otros planteamientos de probabilidades de transición para la rejilla trinomial son los de Tian (1993) y Derman, Kani y Chriss (1996) en donde asignan valor de 1/3 a las probabilidades de transición.

\subsection{Rejillas trinomiales y volatilidad cambiante.}

Siguiendo el modelo propuesto por Haahtela (2010a), trabajar con rejillas trinomiales y volatilidades cambiantes para diferentes periodos, requiere fijar la condición de recombinación de los nodos intemedios. Es menester que se cumpla las siguiente condición:

$$
u \cdot d=m^{2}
$$

La centralidad se encuentra asegurada por la condición $m=e^{r \Delta t}$. La lógica del modelo consiste en calcular probabilidades de transición teniendo como base la mayor volatilidad $\left(\sigma_{\max }\right)$. Los movimientos de ascenso y descenso correspondientes al subyacente se estiman con la máxima volatilidad, partiendo de las siguientes expresiones:

$$
\begin{gathered}
u=e^{r \Delta t+\sqrt{e^{\left(\lambda \sigma_{\max }\right)^{2} \Delta t-1}}} \\
d=e^{r \Delta t-\sqrt{e^{\left(\lambda \sigma_{\max }\right)^{2} \Delta t-1}}} \\
m=e^{r \Delta t}
\end{gathered}
$$

Las probabilidades de transición para el máximo periodo de volatilidad son:

$$
p_{u(\max )}=\frac{m^{2}\left(e^{\sigma_{\max }^{2} \Delta t}-1\right)}{u^{2}+m d-u m-u d}
$$




$$
\begin{aligned}
& p_{d(\max )}=p_{u}\left(\frac{m-u}{d-m}\right) \\
& p_{m(\max )}=1-p_{u}-p_{d}
\end{aligned}
$$

Las probabilidades de transición para los periodos de menor volatilidad se derivan de las ecuaciones anteriores, a partir de proporcionar niveles de volatilidad:

$$
\begin{aligned}
& p_{u}^{i}=p_{u(\max )}\left(\frac{\sigma_{i}}{\sigma_{\max }}\right)^{2} \\
& p_{d}^{i}=p_{d(\max )}\left(\frac{\sigma_{i}}{\sigma_{\max }}\right)^{2} \\
& p_{m}^{i}=1-p_{u}^{i}-p_{d}^{i}
\end{aligned}
$$

La mecánica para la construcción de la rejilla es similar a las ya tratadas en el trabajo: primero se proyecta el proceso estocástico del subyacente para luego introducir los flujos de pagos de la opción en el modelo. La lógica del proceso recursivo es similar al modelo binomial, en este caso explicitada en la ecuación 22.

$$
V_{t-1}=\frac{\boldsymbol{p}_{u}^{i} V_{t, u}+\boldsymbol{p}_{m}^{i} V_{t . m}+\boldsymbol{p}_{\boldsymbol{d}}^{i} V_{t, d}}{e^{r \Delta t}}
$$

\subsection{Modelo trinomial desplazado.}

Para los proyectos del tipo start-ups EBT con sesgos en la distribución de los valores posibles, una alternativa consiste en adaptar el modelo trinomial a partir del binomial desplazado (Haahtela, 2010b; Milanesi, Pesce \& El Alabi, 2014) en adelante BD (Es una adaptación del proceso difusión desplazado empleado en la valoración de derivados financieros (Rubinstein, 1983)). La forma de la función correspondiente a la distribución de posibles valores del mismo es un intermedio entre la normal y lognormal, siendo asimétrica (Es una manera intuitiva y flexible de incorporar potenciales sesgos en los valores proyectados de manera determinística o sensibilizando. Se lo puede incorporar en el conjunto de propuestas donde el modelo binomial es transformado para capturar momentos estocásticos de orden superior (Baliero \& Rosenfeld, 2004)). En este caso, a diferencia de la propuesta de Rubinstein (1983), el parámetro de desplazamiento $(\theta)$ se incorpora fuera del proceso estocástico. La forma de la distribución se descompone en dos partes: (a) probabilística, donde el valor del activo subyacente $\left(V_{\theta}\right)$ sigue un proceso geométrico browniano; (b) parámetro de desplazamiento $(\theta)$ determinístico (Camara $\&$ Chung, 2006). La ecuación que describe el proceso estocástico del subyacente hasta el horizonte $T$ es:

$$
V_{\theta, T}=V_{\theta, 0} e^{\left(\mu-1 / 2 \sigma_{\theta}^{2}\right) T+\sigma_{\theta} \sqrt{T} z}+\theta_{0} e^{\mu t} z \sim N(0,1)
$$

$V_{\theta}$ representa el valor con desplazamiento, siendo la volatilidad desplazada $\sigma_{\theta}$ y $\theta$ constituye el parámetro de desplazamiento $\left(V_{\theta}, \sigma_{\theta}, \theta\right)$. El valor esperado y desvío del subyacente en el horizonte $T$ es:

$$
\begin{aligned}
& V_{\theta t}=\left(V_{\theta, 0}+\theta_{0}\right) e^{\mu t} \\
& \sigma_{\theta}\left(V_{t}\right)=\left|V_{\theta, 0}\right| e^{\mu t} \sqrt{e^{\sigma_{\theta}^{2} t}-1}
\end{aligned}
$$

La variable $V_{\theta}$ es expresada en términos absolutos, debido a que puede asumir valores negativos o positivos. Como en todos los modelos de opciones, el principal insumo para la construcción de la rejilla trinomial proviene de la volatilidad del subyancente, $\left(\sigma_{\theta}\right)$. Para su estimación se parte de los parámetros $V_{\theta}$ y $\theta$ de la ecuación (20), por iteración se obtiene $\sigma_{\theta}$. La restricción es el valor $\sigma_{\theta}\left(V_{t}\right)$. El valor del desvío sesgado $\sigma_{\theta}\left(V_{t}\right)$ se obtiene con análisis de escenarios y simulación. Si la volatilidad es cambiante, los valores $\sigma_{\theta} i\left(V_{t}\right)$ corresponden a cada nivel de volatilidad, obtenidos mediante iteración 
de la ecuación 21. En efecto, se obtienen los valores para la máxima volatilidad desplazada $\sigma_{\theta} \max \left(V_{t}\right)$ y luego las volatilidades correspondientes a cada periodo $\sigma_{\theta} i\left(V_{t}\right)$. La máxima volatilidad permite construir los movimientos del subyacente a través de las ecuaciones 9,10 y 11 .

$$
\begin{aligned}
& u_{\theta}=e^{r \Delta t+\sqrt{e^{\left(\lambda \sigma_{\theta} \max \left(V_{t}\right)\right)^{2}} \Delta t-1}} \\
& d_{\theta}=e^{r \Delta t-\sqrt{e^{\left(\lambda \sigma_{\theta} \max \left(V_{t}\right)\right)^{2}} \Delta t-1}}
\end{aligned}
$$

La centralidad queda asegurada mediante la ecuación $11\left(m=e^{r \Delta t}\right)$. El valor del proyecto para cada periodo de tiempo proyectado en la elaboración de la rejilla trinomial sesgada es:

$$
V_{\theta, u, d, m,(t+1)}=\left(u_{\theta} \cdot V_{\theta, t}+\left|\theta_{0}\right| \cdot e^{r \Delta t}\right) ;\left(m \cdot V_{\theta, t}+\left|\theta_{0}\right| \cdot e^{r \Delta t}\right) ;\left(d_{\theta} \cdot V_{\theta, t}+\left|\theta_{0}\right| \cdot e^{r \Delta t}\right)
$$

El nuevo valor desplazado del proyecto es $V_{\theta, t}$, donde $\theta_{0}$ es el valor correspondiente al parámetro de desplazamiento y $r$ el tipo de interés sin riesgo. Los movimientos $(u, m y d)$ son ajustados, sumando a estos el valor que resulta del producto entre el sesgo capitalizado por los periodos $\left(\left|\theta_{0}\right| \cdot e^{r \Delta t}\right)$.

A partir de los movimientos sesgados obtenidos en las expresiones 22,23 y 24 , y teniendo en cuenta las expresiones 12,13 y 14, se construyen las probabilidades de transición desplazadas:

$$
\begin{aligned}
& p_{u \theta(\max )}=\frac{m^{2}\left(e^{\sigma \theta_{\text {max }}^{2} \Delta t}-1\right)}{u_{\theta}^{2}+m d_{\theta}-u_{\theta} m-u_{\theta} d_{\theta}} \\
& p_{d \theta(\max )}=p_{u \theta}\left(\frac{m-u_{\theta}}{d_{\theta}-m}\right) \\
& p_{m \theta(\max )}=1-p_{u \theta}-p_{d \theta}
\end{aligned}
$$

A continuación, son calculadas las probabilidades intermedias, en donde el ajuste es proporcional a las volatilidades segadas:

$$
\begin{aligned}
& p_{u \theta}^{i}=p_{u \theta(\max )}\left(\frac{\sigma \theta_{i}}{\sigma \theta_{\max }}\right)^{2} \\
& p_{d \theta}^{i}=p_{d \theta(\max )}\left(\frac{\sigma \theta_{i}}{\sigma \theta_{\max }}\right)^{2} \\
& p_{m \theta}^{i}=1-p_{u \theta}^{i}-p_{d \theta}^{i}
\end{aligned}
$$

Finalmente, es aplicado el proceso recursivo para estimar el valor teórico de la opción:

$$
V_{\theta(t-1)}=\frac{p_{u \theta}^{i} V_{\theta t, u \theta}+p_{m \theta}^{i} V_{\theta t . m \theta}+p_{d \theta}^{i} V_{\theta t, d \theta}}{e^{r \Delta t}}
$$

\subsection{Función de utilidad isoelástica y el modelo trinomial desplazado con volatilidad cambiante.}

El uso de funciones de utilidad para valorar los nodos de la rejilla trinomial permite la incorporación del grado de aversión al riesgo del agente, siendo del tipo exponencial isoelásticas las empleadas en rejillas binomiales (Ochoa \& Vasseur, 2014; Pareja \& Cadavid, 2016; Milanesi, 2018). Las funciones de utilidad isoelásticas constituyen un caso especial de la forma hiperbólica de aversión absoluta al riesgo $(H A R A)$. Éstas satisfacen la condiciónes de derivada primera positiva y segunda negativa $\left(U^{\prime}(W)>0\right)$; $\left(U^{\prime \prime}(W)<0\right)$. Se las conoce con las siglas $C R R A$ siendo sus principales características las siguientes las restricciones marcadas por la ecuación 32: 


$$
U(W)=\left\{\begin{array}{c}
\frac{W^{\gamma}-1}{1-\gamma} \rightarrow \gamma>0 ; \gamma \neq 1 \\
\log (W) \rightarrow \gamma=1
\end{array}\right.
$$

En esta $\gamma$ representa el nivel de aversión al riesgo. Cumple con la condición de Inada (conocida por el apellido del economista japonés Ken-Ichi Inada, formulada para la función de producción. El valor de la función es cero en cero; diferenciable en todos sus puntos, creciente en $\mathrm{x}$, de derivada decreciente (cóncava), el límite de la derivada cercana al origen es infinito y el límite de la derivada hacia el infinito positivo es cero). La función de utilidad converge a la logarítmica con gamma tendiendo a 1, con la regla de L'Hopital, donde con $\gamma \rightarrow 1$ numerador y denominador de la función tienden a cero. Al ser diferenciable con respecto a $\gamma$, para tomar el límite de la relación de las derivadas cuando $\gamma \rightarrow 1$, la función de utilidad converge a logarítmica (Ochoa \& Vasseur, 2014). En la condición de Inada se aproxima la utilidad marginal a valores de infinito para riquezas tendientes a cero, sin permitir utilidad con riqueza igual a cero (Suen, 2009) y posibilitando la elasticidad de sustitución intertemporal constante. Esto último como condición que permite asegurar la existencia de equilibrios balanceados (Ljungqvist \& Sargent, 2000). El valor correspondiente a la medida de aversión al riesgo $(\gamma)$ es una variable crucial en la estructura de la ecuación y, al mismo tiempo, es objeto de inumerables calibraciones producto de investigaciones empíricas. No es objeto en el presente trabajo analizar las mismas (éstas se pueden encontrar en Pareja \& Baena, 2018; Chavez, Milanesi \& Pesce, 2019).

En teoría, $\gamma$ debe fluctuar entre -1 y 1 (Pratt, 1964); pero empíricamente, el valor que arroja el coeficiente depende de las características del individuo. Los valores negativos representan personas afectas al riesgo, los positivos adversos y cero corresponde a personas neutrales al riesgo. En el modelo propuesto, la medida de aversión al riesgo se supondrá variable en el tiempo: a mayores horizontes de tiempo, mayor aversión al riesgo $\left(\gamma_{t}\right)$ producto de una mayor incertidumbre. En la expresión 32 el valor correspondiente a la riqueza $(W)$ es sutituido por el valor proyectado del subyacente $V_{\theta, u, d, m,(t+1)}$, obtenido mediante la ecuación 24. Éste es el valor correspondiente al subyacente proyectado con la rejilla trinomial, volatilidad cambiante y sesgo. El grado de aversión al riesgo $\gamma$ se introduce en la función de utilidad (ecuación 32), reformulando la expresión:

$$
U\left(V_{\theta, u, d, m,(t+1)}\right)=\left\{\begin{array}{c}
\frac{\left.V_{\theta, u, d, m,(t+1)}\right)^{\gamma_{t}-1}}{1-\gamma} \rightarrow \gamma>0 ; \gamma_{t} \neq 1 \\
\log \left(V_{\theta, u, d, m,(t+1)}\right) \rightarrow \gamma_{t}=1
\end{array}\right.
$$

La medida de utilidad esperada $E\left[U\left(V_{\theta, u, d, m,(t+1)}\right)\right]$ es obtenida con los coeficientes equivalentes ciertos implícitos sesgados, para cada nivel de probabilidades (ecuaciones 25 a 30). Éstas son aplicadas recursivamente en cada nodo con la lógica de la ecuación 35:

$$
E\left[U\left(V_{\theta, u, d, m,(t+1)}\right)\right]=\left\{\left[p_{u \theta}^{i} U\left(V_{\theta t, u \theta}\right)\right]+\left[p_{m \theta}^{i} U\left(V_{\theta t, m \theta}\right)\right]+\left[p_{d \theta}^{i} U\left(V_{\theta t, d \theta}\right)\right]\right\}
$$

La utilidad esperada permite calcular el coeficiente equivalente cierto. En el caso de que el agente sea neutral al riesgo $(\gamma=0)$, el valor obtenido es el mismo que el que arroja el modelo trinomial con volatilidades cambiantes.

$$
C E\left(V_{\theta, u, d, m,(t+1)}\right)=\left\{E\left[U\left(V_{\theta, u, d, m,(t+1)}\right)\right] \times\left(1-\gamma_{t}\right)\right\}^{\frac{1}{1-\gamma_{t}}}
$$

El equivalente cierto obtenido se actualiza al tipo sin riesgo con la siguiente expresión:

$$
C E\left(V_{\theta, u, d, m,(t)}\right)=C E\left(V_{\theta, u, d, m,(t+1)}\right) \cdot e^{-r}
$$

Cabe destacar que el uso de una tasa libre de riesgo es una simplificación, como oportunamente observa el revisor esto es válido para el precio del subyacente. No lo es para su valor transformado en 
unidades de utilidad. En este caso la tasa de actualización adopta la forma de $r^{*}=(1-$ $\gamma)\left\{r-(\gamma / 2) \sigma^{2}\right\}$

El proceso recursivo brinda una medida monetaria, incorporada en la función de utilidad arroja el nivel de utilidad adaptado al coeficiente de aversión al riesgo del agente. Ésta surge de la siguiente ecuación:

$$
U\left(V_{\theta, u, d, m,(t)}\right)=\left\{\begin{array}{l}
\frac{C E\left(V_{\theta, u, d, m,(t)}\right)^{\gamma_{t}-1}}{1-\gamma} \rightarrow \gamma>0 ; \gamma_{t} \neq 1 \\
\log \left(C E\left(V_{\theta, u, d, m,(t)}\right)\right) \rightarrow \gamma_{t}=1
\end{array}\right.
$$

El modelo trinomial con volatilidad cambiante y aversión al riesgo, si en el extremo: $\bar{\gamma}_{t}=0$ constante (neutralidad al riesgo); $\lambda=1$ (equidistancia entre ascenso y descenso simular a binomial), $\bar{\sigma}_{\theta}$ (volatilidad constante) y $\theta=0$ (inexistencia de sesgo), arroja un resultado coincidente con el modelo binomial clásico.

\section{Funcionamiento del modelo. Análisis de caso.}

En este trabajo no se persigue obtener soluciones estadísticas generalizables, por el contrario, se busca estudiar y poner de manifiesto el comportamiento del conjunto de variables que componen el modelo propuesto y sus interacciones, con el fin de justificar su funcionamiento bajo un determinado paradigma del conocimiento, en este caso, los modelos numéricos de evaluación de activos reales. Como consecuencia de ello, la metodología seleccionada es el estudio de casos en administración. Debe remarcarse que el estudio de casos, entendido como enfoque metodológico, no debe confundirse con el análisis de casos, entendido como un instrumento de enseñanza. La última busca analizar un aspecto concreto relacionado con las organizaciones para fomentar el debate o discusión, mientras que la primera pretende indagar sobre proposiciones teóricas con el objeto de ampliar y generalizar una teoría (Castro, 2010).

El caso a estudiar consiste en un proyecto de inversión en I\&D, con característica distintivas respecto de las inversiones tradicionales, debido al grado de flexibilidad estratégica (opcionalidad) intrínseca generadas por las barreras de entrada (Milanesi, 2018a). Presentan dos características objetivas: a) En los sectores altamente innovadores, los costos de fabricación juegan un rol secundario para definir el precio final del producto, siendo la principal inversión en investigación y desarrollo $(\mathrm{I}+\mathrm{D})$, de ahí que el costo del capital sea uno de los principales factores para explicar el costo del producto. b) Existencia de barreras de entrada siendo una forma de proteger la innovación, a través de patentes, modelos de utilidad, etc.

En este tipo de inversiones el perfil del inversor juega un rol preponderante, pues este tipo de emprendimientos se caracteriza por alta incertidumbre, requerimientos constantes de aportes de capital y desarrollos pautados para horizontes prolongados de tiempo.

Según el perfil del inversor diferenciamos: (a) Inversores aportantes del capital semilla: En esta fase la figura del inversor suele ser el propio emprendedor (investigador o consorcios Agencias de Investigación, Universidades). Mediante el mecanismo de subsidios se obtiene el capital inicial que sirve para financiar los desarrollos de la fase de prototipo y eventualmente las primeras etapas empresariales. (b) Inversores ángel y capital de riesgo: agentes e inversores institucionales que asumen riesgo invirtiendo en la etapa inicial a cambio de participación accionarial, asumiendo un elevado nivel de riesgo. (c) Private equity: inversores institucionales en empresas cerradas maduras y sostenibles en el tiempo. Por lo general la inversión apunta a crear valor en la firma, al detectar un management deficiente o potenciales sinergias en su cartera de negocios. Aportan capital y/o equipo de gerencia. 
Sus etapas son: (a) Etapa preindustrial: madurada la idea, se determina su viabilidad tanto técnica como empresarial, se ingresa en una etapa preindustrial caracterizada por una fuerte aplicación de recursos y una intensa labor de I+D. (b) Lanzamiento al mercado: en caso de que los resultados de la investigación sean positivos, y tras la protección de la innovación generalmente mediante patente, se entra en una fase de comercialización. (c) Etapa de crecimiento: caracterizada generalmente por un fuerte incremento de ingresos, hasta que se llega a un punto de equilibrio en el que se satura el mercado. (d) Etapa de madurez: la duración de este período dependerá del tiempo de explotación de la patente y de la introducción de nuevos productos, servicios y/o tecnologías, que traerán consigo una pérdida de cuota de mercado. (e) Etapa de declive: caracterizada por un decrecimiento de los ingresos, hasta un punto en el que el proyecto/empresa no sea sostenible.

Se supone un emprendimiento de base tecnológica, que se encuentra en su etapa de desarrollo, previéndose un horizonte para la finalización de pruebas y homologación para su lanzamiento en el mercado de 4 periodos $(t=4)$. Se supone un valor actual estimado por el método de descuento de flujos de fondos, ascendiendo a $V_{0}=-\$ 100$ (miles). Tradicionalmente a partir de dicho valor actual esperado se procedía a estimar la volatilidad mediante el enfoque MAD (Copeland \& Antikarov, (2001); Smith, 2005). No obstante, está demostrado el sesgo de la medida en la valoración de la opción, impactando en el valor de la opción. Existen propuestas superadoras al modelo MAD como los trabajos de Brandao, Dyer y Hahnn (2012) y Pareja, Prada y Moreno (2019). En el presente trabajo se supone la volatilidad dada, a los efectos de ilustrar el comportamiento del resto de las variables correspondiente al modelo. Si se considera este valor sin analizar las alternativas estratégicas, debe rechazarse la I\&D del proyecto por carecer de valor económico. No obstante, existen alternativas estratégicas, y conforme fue expuesto, son las que dan sentido y valor a este tipo de proyectos. En el caso bajo estudio, existen dos alternativas ejercibles en $t=4$ : a) desarrollar la etapa de comercialización del producto, con una inversión estimada de $\mathrm{I}=\$ 3.000$ (miles), b) transferir la licencia, con un ingreso de $\$ 9$ (miles) y costo de transferencia de $\$ 2,35$ (miles). La cantidad de pasos para construir la rejilla binomial es de $n=8$, con un $\Delta t=0,5$. La tasa libre de riesgo es del 5\% anual, se supone un sesgo $(\theta)$ en el valor esperado del activo de $|\$ 1.000|$. La rejilla trinomial tiene un coeficiente de dispersión entre nodos $(\lambda)$ de 1,5. El coeficiente de aversión al riesgo se supone cambiante y creciente a medida que el horizonte temporal se hace distante, en consonancia con la mayor incertidumbre percibida por el inversor, siendo de $\gamma_{1}=0,4 ; \gamma_{2}=0,56 ; \gamma_{3}=0,7$; $\gamma_{4}=0,8$; un comportamiento claramente adverso al riesgo.

Para estimar la volatilidad desplazada correspondiente a cada periodo, se parte de las ecuaciones 20 y 21 . La primera sirve para proyectar el valor sesgado del subyacente mediante escenarios y simulación (Milanesi, Pesce \& El Alabi, 2014). Con el valor del desvío estándar sesgado para cada nivel de volatilidad $\sigma_{\theta} i\left(V_{t}\right)$ iterando se obtiene el valor porcentual de $\sigma_{\theta} i$ para la máxima volatilidad y el resto de los volatilidades. En la siguiente tabla se exponen las variables de entrada del modelo.

Tabla 1. variables de entrada del modelo, valor esperado, volatilidad y aversión al riesgo para diferentes horizontes.

\begin{tabular}{|c|c|c|c|c|c|c|}
\hline Periodo & & $\mathbf{0}$ & 1 & 2 & 3 & 4 \\
\hline$V_{\theta t}=\left(V_{\theta, 0}+\theta_{0}\right) e^{\mu t}$ & $\$$ & 900,00 & 946,14 & 994,65 & $1.045,65$ & $1.099,26$ \\
\hline$\sigma_{\theta} i\left(V_{t}\right)$ & $\$$ & - & $\$ \quad 460,00$ & 582,00 & 658,00 & 721,00 \\
\hline$\sigma_{\theta} i$ & & & $46,06 \%$ & $38,37 \%$ & $33,35 \%$ & $29,91 \%$ \\
\hline$\gamma$ & & 0 & 0,40 & 0,56 & 0,7 & 0,8 \\
\hline
\end{tabular}

Fuente: Elaboración propia.

Los valores de la Tabla 1 permiten obtener los coeficientes de ascenso, descenso y medio (ecuaciones 26, 27 y 28), las probabilidades de transición desplazadas de máxima volatilidad (ecuaciones 30,31 y 32 ) y las probabilidades de transición intermedias, proporcionadas a cada nivel de volatilidad (ecuaciones 32, 33 y 34), conforme se expone en la Tabla 2. 
Tabla 2. Parámetros rejilla trinomial desplazada con volatilidad cambiante.

\begin{tabular}{|c|c|c|c|c|}
\hline Periodo & $\mathbf{1}$ & $\mathbf{2}$ & $\mathbf{3}$ & $\mathbf{4}$ \\
\hline$u_{\theta}$ & 1,72329055 & 1,72329055 & 1,72329055 & 1,72329055 \\
\hline$m_{\theta}$ & 1,02531512 & 1,02531512 & 1,02531512 & 1,02531512 \\
\hline$d_{\theta}$ & 0,61003706 & 0,61003706 & 0,61003706 & 0,61003706 \\
\hline$p_{u \theta(\max )}$ & 0,1514269 & & & \\
\hline$p_{m \theta(\max )}$ & 0,5940635 & & & \\
\hline$p_{d \theta(\max )}$ & 0,2545096 & & & \\
\hline$p_{u \theta}^{i}$ & & 0,10506498 & 0,07935907 & 0,06383916 \\
\hline$p_{m \theta}^{i}$ & & 0,71834785 & 0,78725879 & 0,82886367 \\
\hline$p_{d \theta}^{i}$ & & 0,17658717 & 0,13338215 & 0,10729717 \\
\hline
\end{tabular}

Fuente: Elaboración propia.

Las probabilidades de transición se ajustan en función al coeficiente $\lambda$ : a mayor coeficiente, mayor peso cobra $m$, tendiendo a 1 , los extremos $(u \mathrm{y} d)$ tienden a su par binomial. Para proyectar el recorrido del subyacente es utilizada la ecuación 29 , donde primero se proyecta el valor con sesgo y luego éste es extraído. La rejilla queda expuesta de la siguiente manera:

Tabla 3. Valor del proyecto en la rejilla trinomial (elaboración propia)

\begin{tabular}{|c|c|c|c|c|c|c|c|c|c|c|c|c|c|}
\hline & 0 & & 1 & & & & 2 & & & & & & \\
\hline \multirow[t]{17}{*}{$\$$} & $-100,00$ & $\$$ & 525,65 & $\$$ & $1.621,49$ & $\$$ & $3.528,05$ & $\$$ & $6.832,20$ & $\$ 12.545,24$ & $\$ 22.410,01$ & $\$ 39.429,89$ & $\$ 68.780,62$ \\
\hline & & $\$$ & $-102,53$ & $\$$ & 538,95 & $\$$ & $1.662,53$ & $\$$ & $3.617,37$ & $\$ 7.005,16$ & $\$ 12.862,83$ & $\$ 22.977,32$ & $\$ 40.428,06$ \\
\hline & & $\$$ & $-476,28$ & $\$$ & $-105,13$ & $\$$ & 552,60 & $\$$ & $1.704,62$ & $\$ \quad 3.708,94$ & $\$ \quad 7.182,49$ & $\$ 13.188,45$ & $\$ 23.558,99$ \\
\hline & & & & & $-488,34$ & $\$$ & $-107,79$ & $\$$ & 566,59 & $\$ 1.747,77$ & $\$ 3.802,83$ & $\$ 7.364,32$ & $\$ 13.522,32$ \\
\hline & & & & & $-716,34$ & $\$$ & $-500,70$ & $\$$ & $-110,52$ & $\$ \quad 580,93$ & $\$ \quad 1.792,02$ & $\$ \quad 3.899,10$ & $\$ 7.550,75$ \\
\hline & & & & & & & $-734,47$ & $\$$ & $-513,38$ & $\$ \quad-113,31$ & $\$ \quad 595,64$ & $\$ 1.837,38$ & $\$ \quad 3.997,81$ \\
\hline & & & & & & & $-873,56$ & $\$$ & $-753,07$ & $\$ \quad-526,37$ & $\$ \quad-116,18$ & 610,71 & $\$ \quad 1.883,90$ \\
\hline & & & & & & & & & $-895,68$ & $\$ \quad-772,13$ & $\$ \quad-539,70$ & $\$ \quad-119,12$ & $\$ \quad 626,17$ \\
\hline & & & & & & & & & $-980,53$ & $\$ \quad-918,35$ & $\$ \quad-791,68$ & $\$ \quad-553,36$ & $\$ \quad-122,14$ \\
\hline & & & & & & & & & & $\$-1.005,35$ & $\$ \quad-941,60$ & $\$ \quad-811,72$ & $\$ \quad-567,37$ \\
\hline & & & & & & & & & & $\$-1.057,11$ & $\$-1.030,80$ & $-965,44$ & $-832,27$ \\
\hline & & & & & & & & & & & $\$-1.083,87$ & $\$-1.056,90$ & \$ $\quad-989,88$ \\
\hline & & & & & & & & & & & $\$-1.115,45$ & $\$-1.111,31$ & $\$-1.083,65$ \\
\hline & & & & & & & & & & & & $\$-1.143,69$ & $\$-1.139,44$ \\
\hline & & & & & & & & & & & & $\$-1.162,95$ & $\$-1.172,64$ \\
\hline & & & & & & & & & & & & & $\$-1.192,39$ \\
\hline & & & & & & & & & & & & & $\$-1.204,14$ \\
\hline
\end{tabular}

Fuente: Elaboración propia. 
A continuación, en el nodo terminal se debe calcular el valor intrínseco de la opción, para luego aplicar las funciones isoelasticas $U\left(V_{\theta, u, d, m,(t+1)}\right)$; (ecuación 37) en cada nodo. Posteriormente se estima la utilidad esperada con las probabilidades de transición correspondiente a cada nodo $E\left[U\left(V_{\theta, u, d, m,(t+1)}\right)\right]$; (ecuación 38), el equivalente cierto y su valor actual $C E\left(V_{\theta, u, d, m,(t)}\right)$; (ecuaciones 39 y 40). Finalmente y de manera recursiva la utilidad correspondiente al equivalente cierto (ecuación 41). A continuación se presenta el proceso.

Tabla 4. Rejilla trinomial desplazada con volatilidad cambiante y funciones isoelásticas pasos $5,6,7$ y 8.

\begin{tabular}{|c|c|c|c|c|c|c|c|c|c|c|c|c|c|c|c|c|c|c|c|c|}
\hline \multicolumn{12}{|c|}{3} & \multicolumn{9}{|c|}{4} \\
\hline \multicolumn{6}{|c|}{5} & \multicolumn{6}{|c|}{6} & \multicolumn{6}{|c|}{7} & \multicolumn{2}{|r|}{8} & \multirow{2}{*}{ Decisión } \\
\hline$U()$. & & $P V(C E)$ & $C E$ & & $E U()$. & $U()$. & & $P V(C E)$ & $C E$ & & $E U()$. & $U()$. & & $P V(C E)$ & $C E$ & & $U()$. & $U($ (.) & $\$$ & \\
\hline 30,35 & $\$$ & $1.576,93$ & 1616,85 & $\$$ & 30,58 & 35,55 & $\$$ & $2.670,65$ & 2738,25 & $\$$ & 35,82 & 40,69 & $\$$ & $35.690,90$ & 36594,42 & $\$$ & 40,89 & 45,98 & 65780,62 & comercialización \\
\hline 24,80 & $\$$ & 804,44 & 824,81 & $\$$ & 24,99 & 30,93 & $\$$ & $1.678,42$ & 1720,91 & $\$$ & 31,16 & 36,06 & $\$$ & $19.523,11$ & 20017,34 & $\$$ & 36,25 & 41,08 & 37428,06 & comercialización \\
\hline 17,17 & $\$$ & 236,15 & 242,13 & $\$$ & 17,30 & 25,59 & $\$$ & 892,74 & 915,34 & $\$$ & 25,78 & 31,48 & $\$$ & $9.887,92$ & 10138,23 & $\$$ & 31,63 & 36,44 & 20558,99 & comercialización \\
\hline 9,21 & $\$$ & 29,65 & 30,40 & $\$$ & 9,28 & 17,91 & $\$$ & 271,90 & 278,78 & $\$$ & 18,05 & 26,38 & $\$$ & $4.085,45$ & 4188,87 & $\$$ & 26,51 & 31,87 & 10522,32 & comercialización \\
\hline 7,33 & $\$$ & 13,83 & 14,18 & $\$$ & 7,38 & 8,75 & $\$$ & 24,99 & 25,62 & $\$$ & 8,82 & 18,90 & $\$$ & 771,94 & 791,48 & $\$$ & 19,00 & 26,95 & 4550,75 & comercialización \\
\hline 7,16 & $\$$ & 12,81 & 13,13 & $\$$ & 7,22 & 7,28 & $\$$ & 13,49 & 13,83 & $\$$ & 7,33 & 8,07 & $\$$ & 10,93 & 11,21 & $\$$ & 8,11 & 19,90 & 997,81 & comercialización \\
\hline 7,16 & $\$$ & 12,78 & 13,10 & $\$$ & 7,21 & 7,21 & $\$$ & 13,10 & 13,44 & $\$$ & 7,27 & 7,27 & $\$$ & 6,49 & 6,65 & $\$$ & 7,30 & 7,30 & 6,65 & comercialización \\
\hline 7,16 & $\$$ & 12,78 & 13,10 & $\$$ & 7,21 & 7,21 & $\$$ & 13,10 & 13,44 & $\$$ & 7,27 & 7,27 & $\$$ & 6,49 & 6,65 & $\$$ & 7,30 & 7,30 & 6,65 & comercialización \\
\hline 7,16 & $\$$ & 12,78 & 13,10 & $\$$ & 7,21 & 7,21 & $\$$ & 13,10 & 13,44 & $\$$ & 7,27 & 7,27 & $\$$ & 6,49 & 6,65 & $\$$ & 7,30 & 7,30 & 6,65 & transferencia \\
\hline 7,16 & $\$$ & 12,78 & 13,10 & $\$$ & 7,21 & 7,21 & $\$$ & 13,10 & 13,44 & $\$$ & 7,27 & 7,27 & $\$$ & 6,49 & 6,65 & $\$$ & 7,30 & 7,30 & 6,65 & transferencia \\
\hline \multirow[t]{7}{*}{7,16} & $\$$ & 12,78 & 13,10 & $\$$ & 7,21 & 7,21 & $\$$ & 13,10 & 13,44 & $\$$ & 7,27 & 7,27 & $\$$ & 6,49 & 6,65 & $\$$ & 7,30 & 7,30 & 6,65 & transferencia \\
\hline & & & & & & 7,21 & $\$$ & 13,10 & 13,44 & $\$$ & 7,27 & 7,27 & $\$$ & 6,49 & 6,65 & $\$$ & 7,30 & 7,30 & 6,65 & transferencia \\
\hline & & & & & & 7,21 & $\$$ & 13,10 & 13,44 & $\$$ & 7,27 & 7,27 & $\$$ & 6,49 & 6,65 & $\$$ & 7,30 & 7,30 & 6,65 & transferencia \\
\hline & & & & & & & & & & & & 7,27 & $\$$ & 6,49 & 6,65 & $\$$ & 7,30 & 7,30 & 6,65 & transferencia \\
\hline & & & & & & & & & & & & 7,27 & $\$$ & 6,49 & 6,65 & $\$$ & 7,30 & 7,30 & 6,65 & transferencia \\
\hline & & & & & & & & & & & & & & & & & & 7,30 & 6,65 & transferencia \\
\hline & & & & & & & & & & & & & & & & & & & 6,65 & transferencia \\
\hline
\end{tabular}

Fuente: Elaboración propia.

Tabla 5. Rejilla trinomial desplazada con volatilidad cambiante y funciones isoelásticas pasos 1, 2, 3 y 4.

\begin{tabular}{|c|c|c|c|c|c|c|c|c|c|c|c|c|c|c|c|c|c|c|c|c|c|c|c|}
\hline \multicolumn{12}{|c|}{1} & \multicolumn{12}{|c|}{2} \\
\hline \multicolumn{6}{|c|}{1} & \multicolumn{6}{|c|}{2} & \multicolumn{6}{|c|}{3} & \multicolumn{6}{|c|}{4} \\
\hline $\mathrm{U}()$. & \multicolumn{2}{|c|}{$\mathrm{PV}(\mathrm{CE})$} & $\mathrm{CE}$ & \multicolumn{2}{|c|}{$\mathrm{EU}()}$. & \multirow{2}{*}{$\begin{array}{l}\mathrm{U}(.) \\
9,99\end{array}$} & \multicolumn{2}{|c|}{ PV (CE) } & $\mathrm{CE}$ & \multicolumn{2}{|r|}{$\mathrm{EU}()}$. & \multirow{2}{*}{$\begin{array}{c}\mathrm{U}(.) \\
15,79\end{array}$} & \multicolumn{2}{|c|}{ PV (CE) } & $\mathrm{CE}$ & \multicolumn{2}{|r|}{$\mathrm{EU}()}$. & \multirow{2}{*}{$\begin{array}{c}\mathrm{U}(.) \\
23,78\end{array}$} & \multicolumn{2}{|c|}{ PV (CE) } & \multirow{2}{*}{$\begin{array}{c}\mathrm{CE} \\
212,88\end{array}$} & \multicolumn{2}{|c|}{$\mathrm{EU}()}$. \\
\hline 7,65 & $\$$ & 12,67 & 12,99 & $\$$ & 7,76 & & $\$$ & 19,78 & 20,28 & $\$$ & 10,14 & & $\$$ & 81,93 & 84,00 & $\$$ & 15,97 & & $\$$ & 207,63 & & $\$$ & 24,04 \\
\hline 6,90 & $\$$ & 10,67 & 10,94 & $\$$ & 7,00 & 7,55 & $\$$ & 7,55 & 7,74 & $\$$ & 7,74 & 9,83 & $\$$ & 27,87 & 28,58 & $\$$ & 9,94 & 16,39 & $\$$ & 89,11 & 91,37 & $\$$ & 16,57 \\
\hline \multirow[t]{4}{*}{6,75} & $\$$ & 10,29 & 10,55 & $\$$ & 6,85 & 6,93 & $\$$ & 6,93 & 7,11 & $\$$ & 7,11 & 7,51 & $\$$ & 15,12 & 15,50 & $\$$ & 7,59 & 9,61 & $\$$ & 26,50 & 27,17 & $\$$ & 9,72 \\
\hline & & & & & & 6,84 & $\$$ & 6,84 & 7,01 & $\$$ & 7,01 & 7,05 & $\$$ & 13,12 & 13,45 & $\$$ & 7,13 & 7,42 & $\$$ & 14,70 & 15,07 & $\$$ & 7,50 \\
\hline & & & & & & 6,83 & $\$$ & 6,83 & 7,00 & $\$$ & 7,00 & 7,01 & $\$$ & 12,92 & 13,24 & $\$$ & 7,08 & 7,10 & $\$$ & 13,32 & 13,66 & $\$$ & 7,18 \\
\hline & & & & & & & & & & & & 7,00 & $\$$ & 12,91 & 13,23 & $\$$ & 7,08 & 7,08 & $\$$ & 13,23 & 13,57 & $\$$ & 7,16 \\
\hline
\end{tabular}




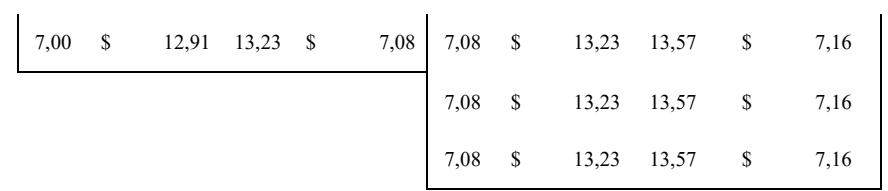

Fuente: Elaboración propia.

Tabla 6. Valor del proyecto: rejilla trinomial desplazada con volatilidad cambiante y funciones isoelásticas.

\begin{tabular}{|c|cc|c|rc|}
\hline \multicolumn{6}{|c|}{ Valor actual } \\
\hline \multicolumn{5}{|c|}{0} \\
\hline $\mathrm{U}()$. & $\mathrm{PV}(\mathrm{CE})$ & $\mathrm{CE}$ & \multicolumn{2}{c|}{$\mathrm{EU}()}$. \\
\hline 6,83 & $\$$ & 10,60 & 10,87 & $\$$ & 6,97 \\
\hline
\end{tabular}

Fuente: Elaboración propia.

El análisis de sensibilidad expone las variaciones en el valor, en este caso, con modificaciones en el sesgo y en la amplitud de los movimientos correspondientes a la rejilla binomial.

Tabla 7. Análisis de sensibilidad, sesgo y amplitud de movimientos.

\begin{tabular}{|c|c|c|c|c|c|c|c|c|c|c|}
\hline 10,60 & \multicolumn{2}{|c|}{ 0 } & \multicolumn{2}{|r|}{-500} & \multicolumn{2}{|c|}{-1000} & \multicolumn{2}{|c|}{-1500} & \multicolumn{2}{|c|}{-2000} \\
\hline 1 & $\$$ & 10,01 & $\$$ & 10,04 & $\$$ & 10,42 & $\$$ & 11,27 & $\$$ & 12,84 \\
\hline 1,5 & $\$$ & 9,99 & $\$$ & 10,05 & $\$$ & 10,60 & $\$$ & 10,83 & $\$$ & 12,88 \\
\hline 2 & $\$$ & 9,98 & $\$$ & 10,07 & $\$$ & 10,75 & $\$$ & 11,20 & $\$$ & 11,41 \\
\hline 2,5 & $\$$ & 9,98 & $\$$ & 10,00 & $\$$ & 10,36 & $\$$ & 10,44 & $\$$ & 13,26 \\
\hline 3 & $\$$ & 9,98 & $\$$ & 10,06 & $\$$ & 10,10 & $\$$ & 11,96 & $\$$ & 12,51 \\
\hline
\end{tabular}

Finalmente, se presentan los diferentes valores, a partir del binomial tradicional, transitando por rejillas trinomiales con volatilidad constante, con y sin sesgo, trinomiales con volatilidad cambiante y trinomiales con volatilidad, sesgo y función isoelástica de utilidad.

En la Tabla 8 se puede apreciar el efecto del sesgo, ya que su inexistencia hace que la opción de continuar no sea viable, por ende los valores positivos se encuentran en la transferencia, arrojando similares resultados el binomial y tradicional $(\$ 5,44 / \mathrm{U}(5,44))$ al asignarse un parámetro $\lambda=1$. Con sesgo, existe posibilidad de continuar y ello es capturado en el resultado $(\$ 47,35 / \mathrm{U}(47,35))$. Si el parámetro $\lambda>1$, entonces binomial y trinomial no coinciden, arrojando el último un mayor valor dada la distribución en tres nodos $(\$ 56,79 / \mathrm{U}(56,79))$. Finalmente incorporando volatilidad variable el resultado se ajusta a la baja producto de los niveles de dispersión menores, quitando peso ponderado a las probabilidades ascendentes $(\$ 31,96 / \mathrm{U}(31,96))$. Finalmente, al incorporar aversión al riesgo variable y creciente en el tiempo, el valor se ajusta a la percepción de un sujeto adverso en mercado incompletos, con sesgos y riesgos variables $(\$ 6,83 / \mathrm{U}(10,6))$. 
Tabla 8. Valores según parámetros.

\begin{tabular}{|c|c|c|c|c|c|c|}
\hline \multirow{2}{*}{ Parámetros } & $\begin{array}{c}\text { Binomial } \\
\sigma=\mathrm{k} ; \theta=0 ; \\
\gamma=0 ; \lambda=1\end{array}$ & $\begin{array}{c}\text { Trinomial } \\
\sigma=\mathrm{k} ; \theta=0 ; \\
\gamma=0 ; \lambda=1,5\end{array}$ & $\begin{array}{c}\text { Trinomial } \\
\sigma=\mathrm{k} ; \theta=0 ; \\
\gamma=0 ; \lambda=1,5\end{array}$ & $\begin{array}{c}\text { Trinomial } \\
\sigma=\mathrm{v} ; \theta=-1000 ; \\
\gamma=0 ; \lambda=1,5\end{array}$ & $\begin{array}{c}\text { Trinomial } \\
\sigma=\mathrm{v} ; \theta=-1000 ; \\
\gamma=0 ; \lambda=1,5\end{array}$ & $\begin{array}{c}\text { Trinomial } \\
\sigma=\mathrm{v} ; \theta=-1000 ; \\
\gamma=\mathrm{v} ; \lambda=1,5\end{array}$ \\
\hline$\theta$ & 0 & 0 & -1000 & -1000 & -1000 & -1000 \\
$\lambda$ & 1 & 1 & 1 & 1,5 & 1,5 & 1,5 \\
$\sigma$ & $\sigma$ (constante) & $\sigma($ constante) & $\sigma($ constante) & $\sigma($ constante) & $\sigma($ variable) & $\sigma$ (variable) \\
$\gamma$ & 0 & 0 & 0 & 0 & 0 & $\gamma($ variable) \\
$\mathrm{U}()$. & 5,44 & 5,44 & 47,35 & 56,79 & 31,96 & 6,83 \\
$\mathrm{PV}(\mathrm{CE})$ & 5,44 & 5,44 & 47,35 & 56,79 & 31,96 & 10,6 \\
\hline
\end{tabular}

Fuente: Elaboración propia.

\section{Conclusiones.}

Frente al no cumplimiento de los supuestos tradicionales relativos a mercados eficientes, perfectos, completos y desarrollados, se deben capturar y valorar tales imperfecciones en el modelo. El modelo binomal y trinomial tradicional con neutralidad al riesgo, supone inversores racionales ilimitadamente, capaces de construir carteras réplicas para estimar el riesgo de los flujos de fondos del proyecto, en especial para inversiones estables cuya volatilidad se mantenga en determinados rangos. Además se requiere de un contexto caracterizado por mercados financieros perfectos, eficientes y completos, en donde prácticamente todos los riesgos son considerados "de mercado" en los términos de Smith y Nau (1995).

No obstante, los mercados emergentes, no completos y en particular proyectos del tipo I\&D o EBT no ven reflejados sus potenciales riesgos. Es allí donde el modelo debe indagar y brindar herramientas al evaluador, que permitan conjugar la ambigüedad del mercado en relación a datos para estimar riesgo, las características dinámicas de la inversión en cuestión y las preferencias del inversor. El modelo propuesto toma las rejillas trinomiales como una herramienta que logra mayor detalle en el mapeo de la granularidad de la rejilla, usa los escenarios y la simulación como técnica para estimar los posibles valores y el sesgo del proyecto, abarcando todos los riesgos del mismo e incorpora las funciones isoeláticas de utilidad incorporando el grado de aversión al riesgo del evaluador.

Esto último de vital importancia, pues no debe perderse de vista que un proceso de valoración es una actividad intelectual donde el conocimiento del evaluador se transforma en parámetro que resulta en valor, que luego toma forma de precio a través del acuerdo de partes y perfeccionamiento de la transacción.

\section{Referencias}

Amram, M., \& Kulatilaka, N. (1998). Real Options (1 ${ }^{\text {a }}$ ed.). Boston, Masachussets, USA: Harvard Business School Prees.

Baliero, R., \& Rosenfeld, R. (2004). Testing Option Pricing with Edgeworth Expansion. Physica A: Statistical Mechanis an its Application, 344, 484-490.

Boyle, P. (1988). A lattice framework for option pricing with two state variables. Journal of Finance and Quantitative Analysis, 23(1), 1-12. 
Brandao, L., \& Dyer, J. (2009). Projetos de Opcoes Reis com Incertezas Correlacionadas. Revista de Administracao e Contabilidade da Unisinos, 6(1), 19-26.

Brandao, L., Dyer, J., \& Hahnn, W. (2012). Volatility estimation for stochastic project value models. European Journal of Operational Research, 220(3), 642-648.

Camara, A., \& Chung, S. (2006). Option Pricing for the Transformed-Binomial Class. Journal of Futures Markets, 26(8), 759-787.

Castro, E. (2010). El estudio de casos como metodología de investigación y su importancia en la dirección y administración de empresas. Revista Nacional de Administración, 2(1), 31-54.

Chance, D. (2007). A Synthesis of Binomial Option Pricing Models for Lognormally Distributed Assets. SSRN: https://ssrn.com/abstract=969834

Chavez, E., Milanesi, G., \& Pesce, G. (2019, septiembre). Estimación de la Aversión al Riesgo Implícita en los precios de mercado de diferentes activos financieros en el mercado argentino. XIX International Finance Conference. http://internationalfinanceconference.org/archive/ifc2019_papers/77.pdf

Copeland, T., \& Antikarov, V. (2001). Real Options (1 ${ }^{\mathrm{a}}$ ed.). New York, USA: Texere LLC.

Cox, J., Ross, S., \& Rubinstein, M. (1979). Option Pricing: A Simplified Approach. Journal of Financial Economics, 7(3), 229-263.

Derman, E., Kani, I., \& Chriss, N. (1996). Implied Trinomial Trees of the Volatility Smile. Quantitative strategies research notes. New York: USA. Goldmand Sachs.

Graeme, G. (2009). Real Options in Theory and Practice (Financial Management Association Survey and Synthesis). Oxford: Oxford University Press.

Guthrie, G. (2011). Learning Options and Binomial Trees. Wilmott Journal, 3(1), 1-23.

Haahtela, T. (2010a). Displaced Diffusion Binomial Tree for Real Option Valuation. SSRN http://dx.doi.org/10.2139/ssrn.1932408

Haahtela, T. (2010b). Recombining trinomial tree for real option valuation with changing volatility. 14th Annual International Conference on Real Options. http://dx.doi.org/10.2139/ssrn.1932411

Hull, J. (2012). Options, Futures and other Derivatives (Global Edition). London, United Kingdom: Pearson Education Limited.

Jabbour, G., Kramin, M., \& Young, S. (2001). Two-state Option Pricing: Binomial Models Revisited. Journal of Futures Markets, 21(11), 987-1001.

Jarrow, R., \& Rudd, A. (1982). Aproximate option valuation for arbitrary stochastic processes. Journal of Financial Economics, 10(3), 347-369.

Kamrad, B., \& Ritchken, P. (1991). Multinomial Approximating Models for Options with k State Variables. Management Science, 37(12), 1640-1653.

Ljungqvist, L., \& Sargent, T. (2000). Recursive Macroeconomic Theory. Massachussetts, USA: MIT press. 
Milanesi, G. (2018). Opciones reales y funciones isoelásticas: el caso de la valuación de un proyecto de I\&D en mercados incompletos. Revista Española de Capital de Riesgo, 2, 39-52.

Milanesi, G., Pesce, G., \& El Alabi, E. (2014). Valoración de empresas de base tecnológica: Análisis de riesgo y el modelo binomial desplazado. Revista Española de Capital de Riesgo, 4, 15-24.

Num, J. (2015). Real options analysis: tools and techniques for valuing strategic investments and decisions with integrated risk management and advanced quantitative decision Analytics $\left(3^{\mathrm{a}}\right.$ ed.). California, USA: ROV.Press.

Ochoa, C., \&Vasseur, J. (2014). Valoración de opciones a través de equivalentes a certeza. Ecos de Economía, 18(39), 49-72.

Pareja, J., \& Baena, J. (2018). Estimación del índice de aversión al riesgo utilizando la función CRRA mediante un diseño experimental. Revista Espacios, 39(13), 29-47.

Pareja, J., \& Cadavid, C. (2016). Valoración de patentes farmacéuticas a través de opciones reales: equivalentes de certeza y función de utilidad. Contaduria y Administración, 61, 794-814.

Pareja, J., Prada, M., \& Moreno, M. (2019). Volatilidad en Opciones Reales: Revisión literaria y un caso de aplicación al sector petrolero colombiano. Revista de Métodos Cuantitativos para la Economía y la Empresa, 27, 136-155.

Pratt, J. (1964). Risk Aversion in the Small and in the Large. Econometrica, 32(1-2), 122-136.

Rendleman, R., \& Bartter, B. (1979). Two-state Option Pricing. Journal of Finance, 34(5), 1092-1110.

Rubinstein, M. (1983). Displaced Diffusion Option Pricing. Journal of Finance, 38(1), 213-217.

Salahaldin, L. (2016). Real Options as a Tool for Value Creation: Evidence from Sustainable Development and Information Technology Sectors. London, United Kingdom: Wiley-ISTE.

Shockley, R.L. (2006). An Applied Course in Real Options Valuation. Sacramento, USA: Thomson South-Western Finance.

Smit, H., \& Trigeorgis, L. (2004). Strategic Investment: Real Options and Games (1 ${ }^{\mathrm{a}}$ ed.). New Jersey, Estados Unidos: Princeton University Press.

Smith, J. (2005). Alternative Approach for Solving Real Options Problems. Decision Analysis, 2(2), $89-102$.

Smith, J., \& Nau, R. (1995). Valuing Risky Projects: Option Pricing Theory and Decision Anaysis. Management Science, 41(5), 795-816.

Suen, R. (2009). Bounding the CRRA Utility Functions. MPRA papers. https://mpra.ub.unimuenchen.de/13260/1/MPRA_paper_13260.pdf

Tian, Y. (1993). A modified lattice approach to option princing. The Journal of Futures Markets, 13(5), 563-577.

Trigeorgis, L. (1995). Real Options in Capital Investment: Models, Strategies and Applications (1 $\left.{ }^{\mathrm{a}} \mathrm{ed}.\right)$. London, United Kindgon: Praeger.

Trigeorgis, L. (1997). Real Options: Managerial Flexibility and Strategy in Resource Allocations (2 ed.). Cambridge: MIT Press. 
Van der Hoek, J., \& Elliot, R. (2006). Binomial models in Finance. New York, United States: Springer Science.

Whaley, R. (2006). Derivatives, Markets, Valuation and Risk Management. New Jersey: John Wiley \& Sons.

Wilmott, P. (2009). Frequently Asked Questions in Quantitative Finance (Segunda ed.). United Kingdom: John Wiley \& Sons. 